Journal for ImmunoTherapy of Cancer

\title{
Enhancing clinical and immunological effects of anti-PD-1 with belapectin, a galectin-3 inhibitor
}

\author{
Brendan D Curti (D) , ${ }^{1}$ Yoshinobu Koguchi, ${ }^{1}$ Rom S Leidner (D) , ${ }^{1}$ Annah S Rolig, ${ }^{1}$ \\ Elizabeth R Sturgill, ${ }^{1}$ Zhaoyu Sun, ${ }^{1}$ Yaping Wu, ${ }^{1}$ Venkatesh Rajamanickam, ${ }^{1}$ \\ Brady Bernard, ${ }^{1}$ lan Hilgart-Martiszus, ${ }^{1}$ Christopher B Fountain, ${ }^{1}$ George Morris, \\ Noriko Iwamoto, ${ }^{2}$ Takashi Shimada, ${ }^{2}$ ShuChing Chang, ${ }^{3}$ Peter G Traber, ${ }^{4}$ \\ Eliezer Zomer, ${ }^{5} \mathrm{~J}$ Rex Horton, ${ }^{5}$ Harold Shlevin, ${ }^{5}$ William L Redmond (1) ${ }^{1}$
}

To cite: Curti BD, Koguchi Y, Leidner RS, et al. Enhancing clinical and immunological effects of anti-PD-1 with belapectin, a galectin-3 inhibitor. Journal for ImmunoTherapy of Cancer 2021;9:e002371. doi:10.1136/jitc-2021-002371

- Additional material is published online only. To view please visit the journal online (http://dx.doi.org/10.1136/jitc2021-002371).

Accepted 08 March 2021

\section{Check for updates}

(c) Author(s) (or their employer(s)) 2021. Re-use permitted under CC BY-NC. No commercial re-use. See rights and permissions. Published by BMJ.

${ }^{1}$ Earle A. Chiles Research Institute, Providence Cancer Institute, Portland, Oregon, USA ${ }^{2}$ Shimadzu Bioscience Research Partnership, Shimadzu Scientific Instruments, Bothell, Washington, USA

${ }^{3}$ Medical Data Research Center, Providence St Joseph Health,

Portland, Oregon, USA

${ }^{4}$ University of Pennsylvania

Perelman School of Medicine,

Philadelphia, Pennsylvania, USA

${ }^{5}$ Galectin Therapeutics,

Norcross, Georgia, USA

\section{Correspondence to} William L Redmond; william.redmond@providence. org

\section{ABSTRACT}

Background PD-1/PD-L1 engagement and overexpression of galectin-3 (Gal-3) are critical mechanisms of tumor-induced immune suppression that contribute to immunotherapy resistance. We hypothesized that Gal-3 blockade with belapectin (GR-MD-02) plus antiPD-1 (pembrolizumab) would enhance tumor response in patients with metastatic melanoma (MM) and head and neck squamous cell carcinoma (HNSCC).

Methods We performed a phase I dose escalation study of belapectin+pembrolizumab in patients with advanced MM or HNSCC (NCT02575404). Belapectin was administered at 2, 4, or $8 \mathrm{mg} / \mathrm{kg}$ IV 60 min before pembrolizumab ( $200 \mathrm{mg}$ IV every 3 weeks for five cycles). Responding patients continued pembrolizumab monotherapy for up to 17 cycles. Main eligibility requirements were a functional Eastern Cooperative Oncology Group status of 0-2, measurable or assessable disease, and no active autoimmune disease. Prior T-cell checkpoint antibody therapy was permitted.

Results Objective response was observed in 50\% of MM (7/14) and and $33 \%$ of HNSCC (2/6) patients. Belapectin+pembrolizumab was associated with fewer immune-mediated adverse events than anticipated with pembrolizumab monotherapy. There were no dose-limiting toxicities for belapectin within the dose range investigated. Significantly increased effector memory T-cell activation and reduced monocytic myeloid-derived suppressor cells (M-MDSCs) were observed in responders compared with non-responders. Increased baseline expression of Gal$3+$ tumor cells and PD-1+CD8+ T cells in the periphery correlated with response as did higher serum trough levels of pembrolizumab.

Conclusions Belapectin+pembrolizumab therapy has activity in MM and HNSCC. Increased Gal-3 expression, expansion of effector memory T cells, and decreased M-MDSCs correlated with clinical response. Further investigation is planned.

\section{INTRODUCTION}

Immune checkpoint inhibitors (eg, aCTLA-4, aPD-1, and aPD-L1 mAb) are approved for the treatment of a variety of metastatic cancers, but monotherapy infrequently cures patients with advanced solid tumors. ${ }^{1-6}$ Combining immune checkpoint inhibitors, such as aCTLA-4+aPD-1, improves overall response rates and is associated with a higher probability of cure compared with checkpoint monotherapy. However, combination therapy with these two checkpoint inhibitors only generates a $10 \%-12 \%$ probability of complete response (CR) and is associated with a higher frequency of severe immune-mediated adverse events than monotherapy. ${ }^{7-11}$ This highlights a central challenge in immunooncology (I-O) research; specifically, how to identify combinations that will evoke durable clinical responses (ideally durable complete remissions), while minimizing toxicity. ${ }^{12}$ The limited understanding of the molecular mechanisms that account for the effectiveness of I-O therapies limits the rational design of more effective combination approaches, which remains a key and unresolved roadblock in the design of I-O clinical trials.

Galectins are part of a family of carbohydratebinding proteins that have affinity for specific oligosaccharides including the C-type lectins, pentraxins, and other galectins. ${ }^{13}$ Galectin-3 (Gal-3), one of 15 galectin family members, is a secreted beta-galactoside binding protein that regulates cell growth and survival. Due to its ability to promote tumor cell proliferation, migration, and metastasis, Gal-3 has been implicated in cancer progression. ${ }^{14-16}$ Extracellular Gal-3 also has immunosuppressive potential because it promotes M2-macrophage polarization and inhibits T-cell activation. ${ }^{17-19}$ Further, a recent report demonstrated that high levels of tumor-specific Gal-3 in patients with non-small cell lung cancer (NSCLC) correlated with decreased responsiveness to PD-1 blockade. ${ }^{20}$ Given the inhibitory effects of Gal-3, preclinical studies explored whether 
inhibiting Gal-3 restored the function of tumor-reactive $\mathrm{T}$ cells. For example, treatment with a Gal-3 inhibitor restored cytokine production and the cytolytic activity of anergic tumor-reactive $\mathrm{T}$ cells isolated from human tumors, including melanoma. ${ }^{21}$ Furthermore, combined treatment with a Gal-3 inhibitor and anti-PD-L1 checkpoint blockade reduced tumor growth in a murine model of lung cancer. ${ }^{22}$

A novel galectin antagonist (belapectin; GR-MD-02) was generated from naturally occurring carbohydrate polymers using proprietary chemical methods ${ }^{23-25}$ Based on preclinical work showing reversal of hepatic fibrosis in animal models, belapectin is being evaluated in clinical trials for patients with fibrotic disease and cancer. A phase I trial with belapectin was conducted in patients with nonalcoholic steatohepatitis (NASH) and advanced fibrosis (NCT01899859).$^{25}$ A phase II multicenter study in NASH cirrhosis (NCT02462967) demonstrated a decrease in portal pressure associated with a prevention of new esophageal varices in patients who have not yet developed them. These studies supported the antifibrotic activity of belapectin and led to the initiation of a phase $2 b / 3$ study with a primary outcome of preventing esophageal varices in patients with NASH cirrhosis (NCT04365868). In these studies, belapectin appeared safe and well tolerated at doses up to $8 \mathrm{mg} / \mathrm{kg}$ for up to 52 weeks. ${ }^{23}$

Based on our preclinical observations that belapectin treatment increased the efficacy of checkpoint blockade and T-cell agonist immunotherapy (manuscript submitted), we conducted a phase I trial with belapectin plus checkpoint blockade (pembrolizumab) in patients with metastatic melanoma (MM) or head and neck squamous cell carcinoma (HNSCC) (NCT02575404). We evaluated the safety and efficacy of this treatment along with comprehensive immune monitoring to explore potential mechanisms of action and putative biomarkers of response.

\section{RESULTS \\ Trial design}

We conducted a phase I clinical trial to investigate the safety and efficacy of combined belapectin plus pembrolizumab immunotherapy in patients with MM or HNSCC (NCT02575404). The main eligibility criteria were metastatic or unresectable melanoma or HNSCC, ECOG 0-2, life expectancy $>12$ weeks, and no active autoimmune disease. Patients with progression on prior checkpoint blockade therapy and non-cutaneous melanoma were accepted. Further details on patient demographics, sites of disease, and prior therapy are provided in table 1 . The full listing of eligibility criteria is provided in the online supplemental materials. The main objective for this study was to determine a maximum tolerated dose of belapectin that could be administered with pembrolizumab. Secondary objectives included an assessment of immune responses and a characterization of toxicities that could be used to generate hypotheses to inform the

\begin{tabular}{lll}
\hline Table 1 & Clinical characteristics & \\
& Melanoma & HNSCC \\
\hline Patients & 14 & 6 \\
\hline Male/Female (\%) & $10 / 4(71 / 29)$ & $6 / 0(100 / 0)$ \\
\hline Median age (range) & $66(38-45)$ & $61(41-69)$ \\
\hline BRAF mutation (\%) & $2(14)$ & $0(0)$ \\
\hline HPV positive (\%) & $0(0)$ & $1(17)$ \\
\hline Prior BRAF-targeted therapy & 2 & 0 \\
\hline Prior immunotherapy (\%) & $10(71)$ & $2(33)$ \\
\hline Anti-CTLA-4 & $6(43)$ & $1(17)$ \\
\hline Anti-PD-1 & $6(43)$ & $1(17)$ \\
\hline Anti-CTLA-4+anti-PD-1 & $1(7)$ & $0(0)$ \\
\hline Interleukin-2 & $4(29)$ & $0(0)$ \\
\hline Other & $7(50)$ & $0(0)$ \\
\hline Prior chemotherapy & $0(0)$ & $3(50)$ \\
\hline Median \# prior treatments & 4 & 3 \\
\hline Prior radiation & $7(50)$ & $5(83)$ \\
\hline
\end{tabular}

*Most patients received multiple sequential monotherapy and combination immunotherapies.

CTLA-4, cytotoxic T lymphocyte antigen-4; HNSCC, head and neck squamous cell carcinoma; HPV, human papillomavirus; PD1 , programmed cell death.

design of future studies investigating the combination of belapectin+pembrolizumab.

A standard 3+3 design was used with an expansion cohort to treat more patients at the highest tolerated or most immunologically active dose level. Belapectin was given at doses of 2, 4, or $8 \mathrm{mg} / \mathrm{kg}$ IV (dose not adjusted for lean body mass) over 60 min and before the administration of pembrolizumab. Pembrolizumab infusions started $60 \mathrm{~min}$ after completion of belapectin and were given at a fixed dose of $200 \mathrm{mg}$ IV over $30 \mathrm{~min}$ on days 1, 22, 43, 64, and 85. Patients with stable disease (SD), partial response (PR) or CR could receive up to 17 total doses of pembrolizumab with cycles every 3 weeks. We anticipated that the doses of belapectin chosen would have biological activity based on preclinical models and NASH clinical trials. Blood samples for immune response evaluation were obtained at baseline, and at days 22, 43, 64, and 85. Tumor biopsies were obtained at baseline and 14 patients with melanoma and six with HNSCC were assessed for response. Of those, six patients were treated in cohort 1 (belapectin dose of $2 \mathrm{mg} / \mathrm{kg}$ ), three in cohort $2(4 \mathrm{mg} / \mathrm{kg})$, and 11 in cohort 3 (8 $\mathrm{mg} / \mathrm{kg}$ ). Response Evaluation Criteria in Solid Tumors (RECIST) version 1.1 was used to assess tumor responses and Common Toxicity Criteria version 4.1 was used to assess and grade adverse events. We defined responding patients as those with a CR or PR using RECIST criteria and non-responding patients as those with stable or progressive disease. 
Table 2 Toxicity assessment

\begin{tabular}{llll}
\hline & Grade & Grade & Grade \\
Toxicity & $\mathbf{1}$ & $\mathbf{2}$ & $\mathbf{3}$ \\
\hline Rash & 9 & & \\
\hline Fatigue & 7 & 3 & \\
Flu-like symptoms & 3 & & \\
Pruritus & 9 & 2 & \\
Diarrhea & 3 & 2 & \\
Joint pain & 3 & & \\
Hyperglycemia & 5 & & \\
\hline Increased alkaline phosphatase & 4 & & \\
Increased AST (aspartate & 4 & \\
aminotransferase) & & \\
Increased ALT (alanine & 4 & \\
transaminase) & & \\
Transient lymphopenia & 3 & \\
\hline
\end{tabular}

\section{Safety}

Combined belapectin+pembrolizumab therapy was well tolerated and there were no toxicities deemed related or probably related to belapectin. All of the toxicities were deemed related to pembrolizumab and all were grade 1 or 2 . One grade 3 toxicity was reported, which was tumor pain in a patient with HNSCG (table 2). No grade 4 toxicities were observed. One patient required oral steroids for grade 2 cholecystitis. This event was deemed possibly related to pembrolizumab and surgery was not needed for management. This event occurred in a patient with HNSCC who experienced a CR (also see below). There was no clear association between belapectin dose level or cancer diagnosis and the frequency or severity of toxicity. No dose-limiting toxicities (DLT) were observed for belapectin within the dose range investigated. We chose to treat six patients in cohort 1 and 11 patients in cohort 3 (highest planned dose level) in order to obtain more data on immune responses.

\section{Clinical response}

Objective responses were seen at each dose level of belapectin and in both MM and HNSCC patients. An illustration of response in a melanoma patient is given in figure 1. The objective response of all patients assessed at day 85 (after five cycles of belapectin+pembrolizumab)
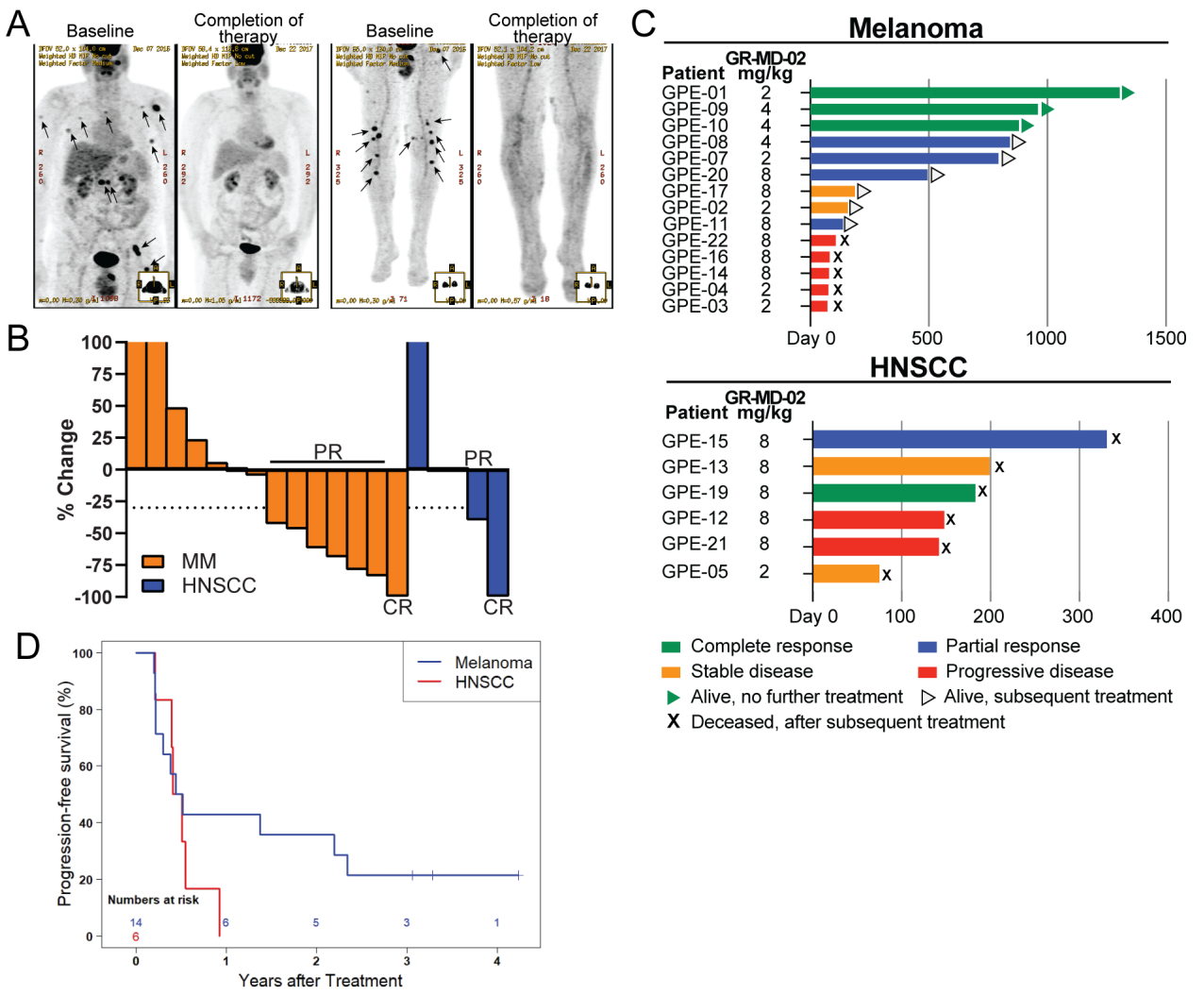

Figure 1 Clinical efficacy of belapectin+pembrolizumab therapy. (A) PET images depict multiple FDG-avid soft tissue lung and LN melanoma deposits at baseline with resolution 1 year after belapectin+pembrolizumab. Baseline images depict extent of melanoma after progression on BRAF/MEK-targeted therapy. (Left panel) Chest, abdomen, and pelvis images. (Right panels) Lower extremity images. Arrows indicate melanoma deposits. (B) Objective response to belapectin+pembrolizumab therapy at day 85 . Graph depicts the $\%$ change in the sum of the diameters of target lesions using RECIST V.1.1 criteria $(n=14$ MM; $n=5$ HNSCC). Dotted line at $-30 \%$ denotes minimum cut-off for partial response. (C) Plot depicts duration of clinical response to belapectin+pembrolizumab therapy. (D) Progression-free survival. CR, complete response; FDG, Fluorodeoxyglucose; HNSCC, head and neck squamous cell carcinoma; LN, lymph node; MM, metastatic melanoma; PET, positron-emission tomography; PR, partial response; RECIST, Response Evaluation Criteria in Solid Tumors. 
is depicted in figure 1B. Among the 7/14 patients with MM who had an objective response to belapectin+pembrolizumab, three had no prior therapy and four had progression after treatment with T-cell checkpoint inhibitors $(\mathrm{n}=2)$, high-dose IL-2 $(\mathrm{n}=1)$, oncolytic virus+aCTLA-4 $(n=1)$, or BRAF-targeted therapy $(n=1)$. One of the MM responders had a mbeucosal (vaginal) primary tumor. Three of the seven responding patients with melanoma have required no further systemic therapy to date (figure 1C). The median progression-free survival (PFS; 95\% CI) was 5.8 (3.6, NA) months in melanoma (figure 1D). Among the 2/6 patients with HNSCC who achieved a response, one had prior progression on T-cell checkpoint blockade therapy (intratumoral ipilimumab, ipi). In addition, 5/6 HNSCC patients were HPV including both responders. The HNSCC patient that achieved a CR died from a metastasis to the gall bladder. It is interesting to note that this was a clinical site of inflammation during treatment. The median PFS $(95 \% \mathrm{CI})$ was 5.5 (4.7, NA) months in HNSCG (figure 1D).

\section{Combination immunotherapy alters systemic immune responses}

We hypothesized that patients with an objective response had higher immune activation following pembrolizumab+belapectin compared with patients who did not achieve objective response. We conducted comprehensive immunological monitoring by measuring changes in circulating leukocytes and serum cytokines and chemokines before and after treatment. We also investigated whether these immune assessments were associated with clinical outcome. Due to variability in the timing of maximal immunologic responses among patients, we selected the peak immune response (between days 22-85 as compared with baseline) as the maximum value for correlation with the best clinical outcome (as determined by RECIST response at day 85 ). We observed a statistically significant increase in proliferating $(\mathrm{Ki}-67+)$ activated (ICOS+) effector memory $\left(\mathrm{CCR}^{-} \mathrm{CD}^{-} \mathrm{RA}^{-}\right) \mathrm{CD} 4+\mathrm{T}$ cells in responding patients (figure 2A). We also detected a statistically significant expansion in the percentage of effector memory CD8+ T cells in responders (figure 2B, top) and increased effector memory T-cell proliferation $(\mathrm{Ki}-67+)$ in responders and non-responders (figure 2B, bottom). We also analyzed regulatory $\left(\mathrm{CD} 25+\mathrm{CD} 127^{\mathrm{lo}}\right)$ CD4+ T-cell responses and found no difference in the frequency of Tregs post-treatment in responders or nonresponders and a similar increase in proliferation (Ki-67+) in both cohorts (online supplemental figure 1 top and middle panels, respectively). There was a significant increase in HLA-DR+ Tregs following treatment in nonresponders only (online supplemental figure 1, bottom panel), which may represent a Treg subset capable of

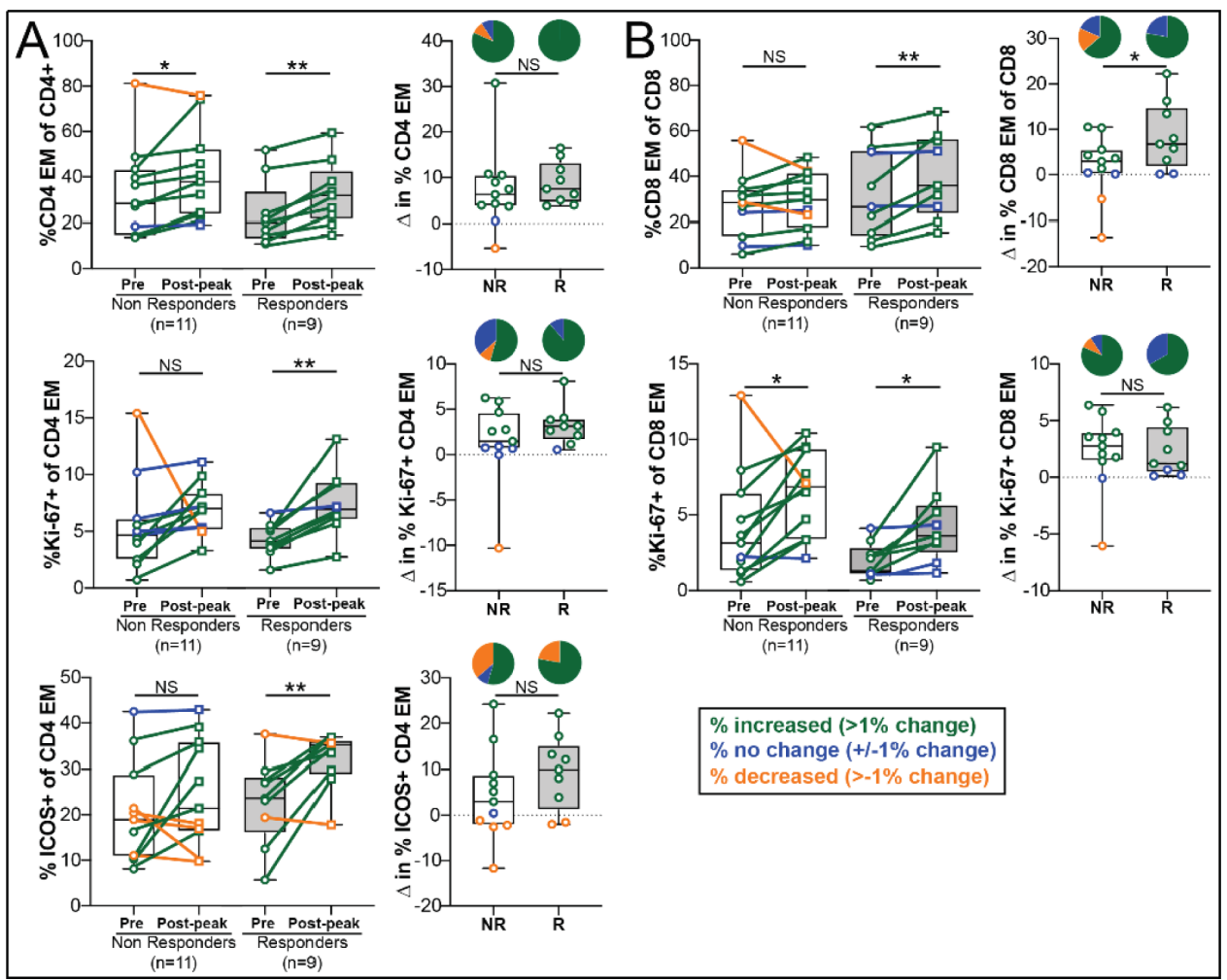

Figure 2 Enhanced T-cell responses following belapectin+pembrolizumab. Fresh whole blood was stained with mAbs to CD45, CD3, CD4, CD8, CD45RA, CCR7, Ki-67, and ICOS. Following RBC lysis and washing, (A) CD4+ and (B) CD8+ T-cell phenotypes were determined by flow cytometry. Each line represents an individual patient and graphs depict the median (center line), the interquartile range (box), and the minimum and maximum values (whiskers) of $n=9-11$ patients per cohort. Green indicates an increase post-treatment versus pretreatment, blue indicates no change, and orange indicates a decrease. ${ }^{*} \mathrm{P}<0.05$, ${ }^{\star \star} \mathrm{p}<0.01$. NR, non-responder (white bars); R, responder (gray bars); RED, red blood cell. 


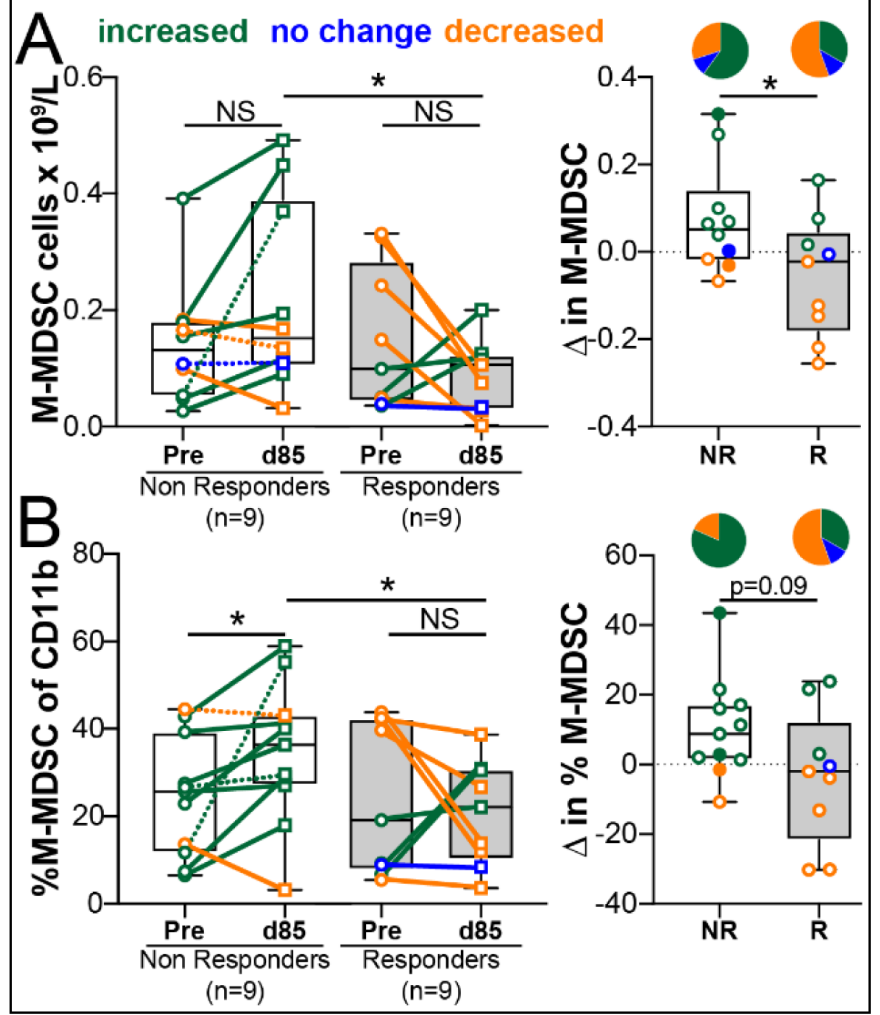

Figure 3 Belapectin+pembrolizumab reduces $M$ -

MDSCs. The (A) total number and (B) percent of M-MDSCs

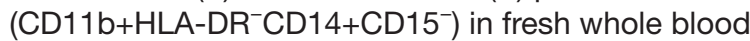
samples at baseline (pretreatment) and day 85 post-treatment were determined by flow cytometry. Each line represents an individual patient and graphs depict the median (center line), the interquartile range (box), and the minimum and maximum values (whiskers) of $n=9$ patients per cohort. Dotted lines (left panels) and filled circles (right panels) in non-responders indicate patients whose final blood draw was before d85 due to disease progression. ${ }^{*} \mathrm{P}<0.05$. M-MDSCs, monocytic myeloid-derived suppressor cells; NR, non-responder (white bars); $\mathrm{R}$ responder (gray bars).

increased contact-dependent suppression. ${ }^{26}$ These data suggest that belapectin+pembrolizumab enhances the activation of effector memory $\mathrm{T}$ cells in responders, along with increasing the percentage of effector memory T-cell proliferation in all patients.

Preclinical models demonstrated that Gal-3 expression affects the polarization, programming, and function of myeloid cells, including monocytes and myeloid-derived suppressor cells (MDSCs), which consist of polymorphonuclear (PMN-MDSC) and monocytic (M-MDSC) subsets. ${ }^{19} 27-32$ Therefore, we investigated the influence of belapectin+pembrolizumab therapy on monocyte and MDSC subsets in the peripheral blood and compared these subsets in responding and non-responding patients. The absolute number (figure 3A) and percent (figure 3B) of M-MDSCs trended lower at day 85 as compared with baseline in responding patients. In contrast, non-responding patients were characterized by a significant increase in the \% M-MDSCs at day 85 as compared with baseline (figure 3B). When we compared the total number and frequency of M-MDSCs at day 85 between responders and non-responders, we found significantly fewer M-MDSCs in responders (figure $3 \mathrm{~A}, \mathrm{~B}$, left panels). Further analysis revealed that six out of nine patients who achieved an objective response showed no change or a decrease in the \% M-MDSC (day 85) compared with baseline, while only two out of nine patients with disease progression showed decreased \%M-MDSC over the same time interval (figure 3B, right panels). In contrast, no differences were observed in the proportion of total monocytes or PMNMDSCs in these patients (data not shown). These data suggest that the majority of responding patients exhibited stabilization or a declining frequency of M-MDSCs as compared with expansion in non-responders, implicating M-MDSCs as a possible biomarker of response and provides insight into the potential mechanism of action of belapectin+pembrolizumab.

Next, we asked whether belapectin+pembrolizumab therapy increased proinflammatory serum cytokine and/ or chemokine levels and whether these cytokines and/ or chemokines also correlated with objective response using RECIST criteria. Forty-five cytokines/chemokines were measured pretreatment and post-treatment and among these analytes, we found statistically significant increases in IP-10/CXCL10, IL-12p70, and IL-18 posttreatment in responders (online supplemental figure 2). These specific proteins are associated with increased T-cell recruitment (IP-10/CXCL10) and proinflammatory (type-1; Th1) immune responses (IL-12, IL-18). ${ }^{33}$ All together, these data provide evidence of an altered systemic immune response including increased T-cell proliferation and proinflammatory cytokines and chemokines, with a concomitant reduction in MDSCs in patients responding to belapectin+pembrolizumab.

\section{Biomarkers of response}

We examined baseline (pretreatment) expression of Gal-3 and PD-L1 in tumor biopsies using multiplex immunohistochemistry (mIHC). We developed two custom seven-color mIHC panels to interrogate the relationship between immune infiltration and Gal-3 expression. Each panel included CD3 (pan T cell), CD8 (cytotoxic T cell), CD163 (macrophage), PD-L1 (immune checkpoint), Gal-3 (biomarker), tumor (SOX10 for melanoma; cytokeratin/CK for HNSCG), and DAPI (nuclei). PD-L1 expression was determined by mIHC following pathology review, which has been demonstrated to have high concordance with the chromogenic assay. ${ }^{34} 35$ In a patient with MM that achieved a PR, we observed high tumor-associated Gal-3 and PD-L1 coexpression (figure 4A), which was representative of other MM specimens. We quantified leukocyte numbers and Gal-3 expression across the entire cohort and asked whether the mIHC data correlated with clinical response. Indeed, patients experiencing clinical benefit (CR/PR) exhibited an increased density of Gal-3+ tumor cells as compared with those with PD (figure 4B, left panel). No trend was seen with regard to stromal CD3+ $\mathrm{T}$ cells and response (figure $4 \mathrm{~B}$, middle panels), while 


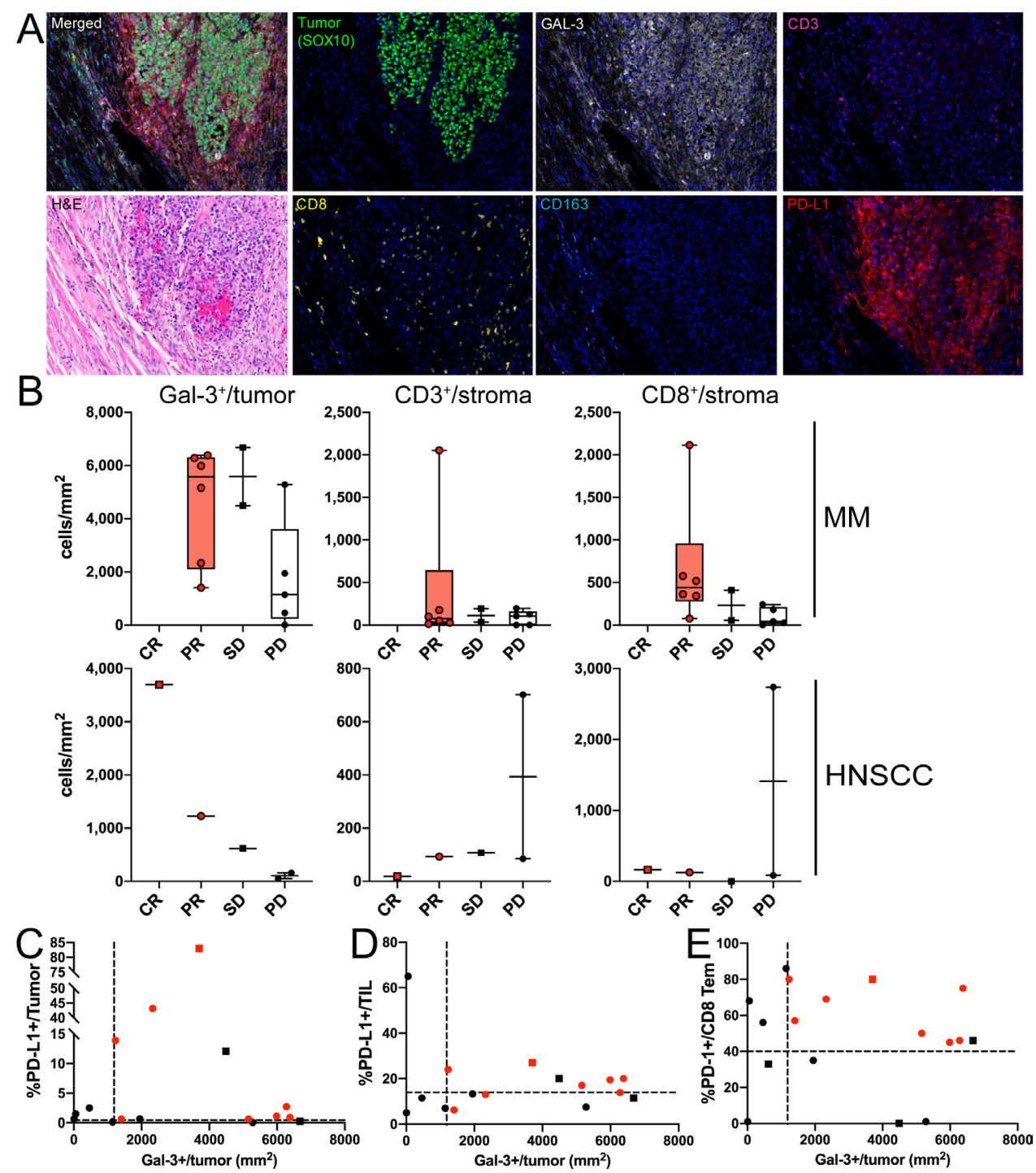

Figure 4 Baseline Gal-3 expression ( $\mathrm{mlHC}$ ) is associated with clinical benefit to belapectin+pembrolizumab therapy. (A) Representative $\mathrm{mIHC}$ from MM pretreatment biopsy (FFPE) that was stained with a seven-color mIHC panel. (B) Graphs depict number of Gal-3+ tumor cells, CD3+ T cells, and CD8+ T cells from $n=13$ (MM) and $n=5$ (HNSCC) patients per cohort, grouped by clinical response. Each point represents one patient. Responder (orange bars); non-responder (white bars). (C-E) Graphs depict (C) PD-L1+ tumor (IHC), (D) PD-L1+ TIL (IHC), or (E) \%PD-1+ CD8+ T cells (flow) versus Gal-3+ tumor (IHC; X-axis) from $n=13(M M)$ and $n=4$ (HNSCC) patients. Each point represents one patient (CR: square; PR: circle; SD: square; PD: circle). Dotted lines represent cut-offs determined by Classification and Regression Tree (CART) analysis (see Table 3). CR,complete response; FFPE, formalin-fixed paraffin-embedded; HNSCC, head and neck squamous cell carcinoma; MM, metastatic melanoma; mIHC, multiplex immunohistochemistry; PET, positron-emission tomography; PD, progressive disease; PR, partial response; RECIST, Response Evaluation Criteria in Solid Tumors; SD, stable disease.

there was a trend of increased stromal CD8+ T cells and response in MM (figure 4B, right panels). Minimal expression of Gal-3 was detected on CD163+ macrophages (data not shown). We also confirmed that tumor PD-L1, but not Gal-3, correlates with the efficacy of aPD-1 monotherapy in melanoma, suggesting that Gal-3 combined with PD-1 expression is a potentially unique biomarker for belapectin+pembrolizumab treatment (online supplemental figure 3).

Increased PD-1+CD8+ $\mathrm{T}$ cells within the TIL and increased T-cell expansion and proliferation (Ki-67) in the peripheral blood have been associated with improved clinical response to checkpoint blockade. ${ }^{36-39}$ Thus, we asked whether baseline expression of Gal-3 and PD-L1 within the tumor identified by $\mathrm{mIHC}$ and/or the extent of PD-1+ T cells in the peripheral blood as determined by flow cytometry could serve as predictive biomarkers of response to belapectin+pembrolizumab. Coexpression of PD-L1 on tumor or immune cells and Gal-3 on tumor cells, as quantified by mIHC, did not predict clinical benefit (figure 4C,D, respectively). In contrast, $100 \%$ of patients achieving an objective response (CR or PR) could be characterized by both the percent of circulating PD-1+ effector memory CD8+ T cells and the extent of Gal-3+ tumor cells present at baseline (figure 4E). Additional analysis demonstrated that PD-1 expression on multiple 
Table 3 Correlation of PD-1+CD8+ T cells (peripheral blood) and Gal-3+ tumor cells with favorable clinical outcomes following belapectin+pembrolizumab immunotherapy

\begin{tabular}{llll}
\hline Group* $^{*}$ & CR/PR & SD/PD & P value† \\
\hline High-risk, n (\%) & $0(0)$ & $8(89)$ & 0.0004 \\
Low-risk, n (\%) & $8(100)$ & $1(11)$ & \\
\hline
\end{tabular}

*Group including high-risk and low-risk subgroups defined by Classification and Regression Tree (CART) analysis: high-risk if \%PD-1+/CD8 Teff < 24 or \%PD-1+/CD8 Teff $>24$ and Gal-3+/ tumor < 1186; low-risk if \%PD-1+/CD8 Teff $>24$ and Gal-3+/tumor $>1186$.

†Fisher exact test.

$\mathrm{CR}$, complete response; $\mathrm{PD}$, progressive disease; $\mathrm{PR}$, partial response; SD, stable disease.

T-cell subsets including central, effector, and effector memory CD4+ and CD8+ T cells plus Gal-3 expression on tumor cells was associated with response (table 3), indicating that patients with an increased frequency of circulating PD-1+T cells at baseline were more likely to respond.

Recent studies have demonstrated an association between reduced clearance (higher trough level) of aPD-1 and better survival in patients with melanoma or NSCLC. ${ }^{40}{ }^{41}$ Thus, we hypothesized that the trough level of pembrolizumab, as assessed by mass spectrometry analysis, may be a biomarker of clinical response to belapectin+pembrolizumab. Patients with disease control $(\mathrm{CR}+\mathrm{PR}+\mathrm{SD})$ at day 85 post-treatment showed
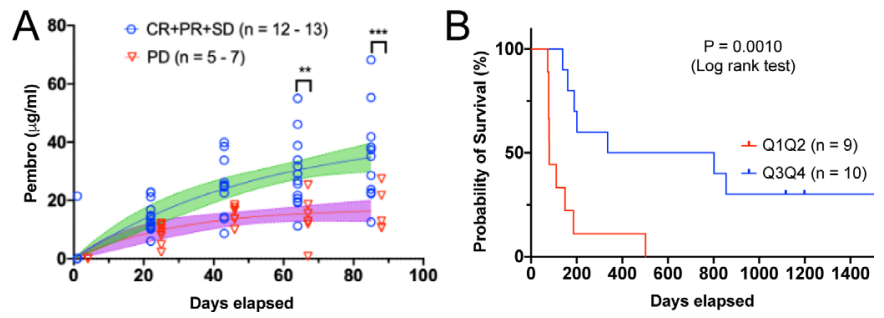

Figure 5 Increased trough levels of pembrolizumab following belapectin+pembrolizumab therapy correlate with improved clinical outcome. (A) Serum trough levels of pembrolizumab are shown for patients with disease control (CR+PR+SD: open blue circles) or progressive disease (PD: open red downward triangles). The $95 \%$ confidence band is depicted for patients with disease control (green) or disease progression (purple). Statistical differences in pembrolizumab concentrations (mean) across patient groups and time points were confirmed by two-way ANOVA, multiple comparisons were done using Sidak's multiple comparisons test, ${ }^{* *} \mathrm{p}<0.01$, ${ }^{* * *} \mathrm{p}<0.001$. (B) Kaplan-Meier curve depicting increased progression-free survival in patients with higher trough level of pembrolizumab. Patients were grouped based on the trough levels of pembrolizumab at day 43: Q1Q2 (red: below population mean) and Q3Q4 (blue: above population mean). Log rank test was used to assess the statistical difference in survival times between the groups. ANOVA, analysis of variance; $\mathrm{CR}$, complete response; $\mathrm{PR}$, partial response; $\mathrm{PD}$, progressive disease; SD, stable disease. higher trough levels of pembrolizumab at days 64 and 85 compared with those with progressive disease (figure 5A). Furthermore, higher pembrolizumab trough levels at day 43 correlated with significantly improved PFS (figure 5B). Together, these data suggest that increased baseline expression of Gal-3 in the tumor, higher aPD-1 trough levels, and increased circulating PD-1+ CD8+ T cells are all potential biomarkers of response.

\section{DISCUSSION}

While aPD-1 monotherapy is used widely in MM, HNSCC, lung cancer, renal cancer, non-melanoma skin cancers, breast cancer, microsatellite instable-high malignancies, and other solid tumors, cures are infrequent, and, depending on the cancer diagnosis and prior lines of therapy, the probability of response is only $20 \%-40 \%$. Currently, aCTLA-4+aPD-1 is the most effective immunotherapy combination for MM and renal cell cancer and has significant activity in other solid tumors including lung cancer. Although combination therapy enhances survival as compared with aCTLA-4 or aPD-1 monotherapy, cures remain infrequent and toxicity is significant. Thus, novel therapeutic strategies, particularly those targeting distinct mechanisms of suppression are needed to improve outcomes. Our preclinical studies revealed that blockade of the immunosuppressive molecule, Gal-3, enhanced the antitumor efficacy of checkpoint inhibitors and T-cell agonists (manuscript submitted). These data provided the rationale for our current phase I clinical trial, in which we explored the safety of a Gal-3 inhibitor (belaptectin; GR-MD-02) plus pembrolizumab in patients with MM or HNSCC.

We observed objective responses in $50 \%$ of patients with advanced and heavily pretreated MM and in $33 \%$ of patients with HNSCC. The objective response rates to pembrolizumab monotherapy in randomized studies in patients with advanced melanoma ranges from $21 \%$ in patients who have had prior therapy to $39.1 \%$ in patients who had not received prior systemic immunotherapy. ${ }^{42}$ Additionally, 3/14 (21.4\%) melanoma patients in this study ultimately achieved a CR (figure 1C), which compares favorably to historical controls. The objective response of pembrolizumab monotherapy in HNSCC cancer is $15 \%{ }^{44-47}$ and while we observed responses in 2/6 (33\%) HNSCC patients, the small sample size in the current study precludes any conclusions regarding the potential increased efficacy of belapectin+pembrolizumab in this setting. There was no significant toxicity from the combination of belapectin+pembrolizumab (table 2). We did not reach DLT of belapectin within the range of doses investigated in this study. We have chosen a belapectin dose of $4 \mathrm{mg} / \mathrm{kg}$ for further study as it was well-tolerated, showed clinical and immunological effects in conjunction with pembrolizumab, and did not add to the adverse events associated with pembrolizumab. In the Keynote006 study, which investigated the outcomes of patients with advanced melanoma after pembrolizumab, $76 \%$ of 
patients had treatment-related adverse events and 12\% of patients had grade 3-5 immune-mediated adverse events compared with $0 \%$ with belapectin+pembrolizumab. ${ }^{48}$ We hypothesize that belapectin therapy may reduce toxicity by decreasing M2 macrophage polarization, thereby reducing inflammation that could lead to immune-mediated hepatic, pulmonary, gastrointestinal, or rheumatological toxicity. We acknowledge the inherent limitations associated with phase I clinical trials including the lack of a control arm and are planning a larger randomized placebo-controlled phase II study comparing belapectin+pembrolizumab with pembrolizumab monotherapy in patients with advanced MM or HNSCC to better define the toxicity, antitumor activity, and immune changes associated with the combination.

Our comprehensive immune monitoring of peripheral blood revealed a significantly increased frequency of effector memory CD4+ and CD8+ T cells and in their activation status (Ki-67+CD4+, ICOS+CD4+, Ki-67+CD8+) in responders following belapectin+pembrolizumab therapy (figure 2). One other report described increased CD8+ T-cell-specific Ki-67 and ICOS expression following aPD-1 therapy. Specifically, the authors found increased $\mathrm{Ki}-67+\mathrm{PD}-1+\mathrm{ICOS}+\mathrm{CD} 8+\mathrm{T}$ cells post-treatment in the peripheral blood of up to $80 \%$ of patients with NSCLC that derived clinical benefit from aPD-1. In contrast, they found reduced or absent CD8+ T-cell responses in 70\% of patients with progressive disease. ${ }^{49}{ }^{50}$ We have additional studies underway to define the effector phenotype and tumor reactivity of the proliferating cells and to determine whether the phenotypes observed among circulating $\mathrm{T}$ cell mirrors the local response within the tumor. Specifically, we plan to investigate the immunological signature (ie, protein, RNA expression) of leukocytes from paired blood/tumor specimens, which will add critical insight into the specificity of the tumor-reactive $\mathrm{T}$ cells as well as potential differences in their transcriptional signature that may be associated with clinical benefit.

We also observed a marked decrease in circulating M-MDSCs within responding patients compared with non-responding patients (figure 3), suggesting that combination therapy limited the expansion of this immunosuppressive population, which can suppress T-cell activation via cytokine secretion (eg, IL-10) and/or metabolic alterations (eg, Arg1) within the tumor microenvironment (TME) ${ }^{29}{ }^{30}$ A correlation between increased levels of MDSCs and worse outcomes has been reported in melanoma patients receiving checkpoint blockade with ipi (aCTLA-4). In these patients, the baseline percentage of circulating MDSCs inversely correlated with response to aCTLA-4 and OS. ${ }^{51-53}$ Limited data exist for the effect of pembrolizumab on MDSCs. One study has shown that the baseline percentage of circulating MDSCs inversely correlated with response and OS in melanoma patients treated with nivolumab. However, all these patients had received CTLA-4 blockade prior to nivolumab, which complicates interpretation of these data. ${ }^{54}$ To the best of our knowledge, the relationship between percent
M-MDSCs and response has not been observed in patients who received pembrolizumab monotherapy. We observed that patients achieving clinical response generally exhibited stable levels or reduced M-MDSCs following pembrolizumab+belapectin treatment, suggesting that belapectin may inhibit immune suppression by M-MDSCs.

In a recent report, high Gal-3 expression correlated with a poor response to PD-1 blockade in a small cohort of NSCLC patients with high baseline PD-L1 expression. ${ }^{20}$ However, reanalysis of tumor RNA-seq data showed that Gal-3 expression did not influence the effect of PD-1 blockade in melanoma (online supplemental figure 3). In our study, we observed a significant correlation between increased tumor-specific Gal-3 expression and baseline PD-1+ T cells and favorable clinical response (figure 4E). We also analyzed serum levels of pembrolizumab posttreatment and observed a correlation between increased trough levels of pembrolizumab and favorable clinical responses in patients treated with belapectin+pembrolizumab therapy (figure 5). These data are consistent with previous reports of the correlation between increased serum trough aPD-1 mAb levels, including pembrolizumab and nivolumab, with clinical response. ${ }^{40} 45$ The association between reduced drug clearance and clinical response is likely a general feature of checkpoint inhibitors and not specific to pembrolizumab. Although the underlying mechanisms remain unclear, there are at least two proposed interpretations for this inverse clearance-response relationship: (1) an exposure-outcome relationship (cause) ${ }^{41}$; and (2) a reflection of an underlying patient-specific condition, such as metabolic state (effect). ${ }^{40} 55-57$ The first possibility (causal relationship) has a clinically important implication because it suggests that increased dosing may improve clinical outcome. However, Turner et al observed the same inverse clearance-response relation in both the $2 \mathrm{mg} / \mathrm{kg}$ and $10 \mathrm{mg} /$ $\mathrm{kg}$ cohorts, suggesting that this is probably not the case. ${ }^{40}$ The second interpretation argues that a patient's metabolic state determines the rate of clearance such that a positive response may improve their metabolic state and, in turn, reduce the rate of clearance. Of note, we did not observe any differences in the distribution of trough pembrolizumab concentrations among the three dosing regimens, suggesting that belapectin did not influence directly the pharmacokinetics of pembrolizumab (data not shown). Together, these data suggest that increased trough levels of pembrolizumab may be a potential biomarker for clinical outcome.

In summary, these data demonstrate that belapectin+pembrolizumab therapy is safe, associated with increased T-cell activation, limited expansion of M-MDSCs, and favorable clinical responses. We propose that in the absence of therapy, PD-L1 and Gal-3 contribute to M2-macrophage polarization and reduced CD8+ T-cell recruitment to the tumor site (figure 6 ). In the presence of PD-1 blockade, CD8+ T cells can be reinvigorated, but suppressed due to other inhibitory signals. In contrast, combination belapectin+pembrolizumab therapy enables 


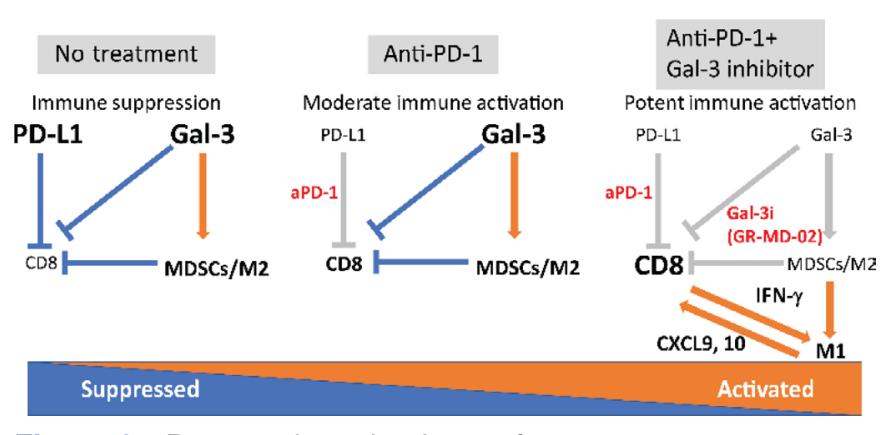

Figure 6 Proposed mechanisms of belapectin+pembrolizumab therapy. The immunosuppressive TME impedes CD8+ T cells through PD-1/PD-L1 and Gal3 (left). Anti-PD-1 alleviates PD-L1 suppression, but Gal-3 still inhibits the effector function of CD8+ T cells, resulting in limited immune activation (middle). Dual PD-L1 and Gal3 blockade boosts CD8+ T cells, reduces MDSCs, and repolarizes $\mathrm{M} 2 \rightarrow \mathrm{M} 1$ macrophages, augmenting antitumor immunity (right). Belapectin (GR-MD-02), galectin-3 inhibitor; Gal-3, galectin-3; MDSCs, myeloid-derived suppressor cells; TME, tumor microenvironment.

T-cell activation in the presence of a more proinflammatory TME capable of supporting T-cell effector function and recruitment, and subsequently tumor regression. Most of the patients who volunteered for this study had progression after CTLA-4 and/or PD-1 blockade, BRAFtargeted therapy, or chemoradiotherapy. In this group of heavily pretreated patients, we report durable objective responses with toxicity that was less than anticipated with pembrolizumab monotherapy. Comprehensive immune profiling revealed candidate biomarkers of immune response including tumor-specific Gal-3 expression and PD-1+ effector memory $\mathrm{T}$ cells that appeared to enrich for patients more likely to respond to belapectin+pembrolizumab treatment. Furthermore, mass spectrometry analysis revealed that trough levels of pembrolizumab in the serum could also serve as a potential biomarker for clinical outcome. These data provide a strong rationale to perform a randomized placebo-controlled phase II clinical trial to evaluate the efficacy and immune changes of belapectin+pembrolizumabversus pembrolizumab in patients with MM or HNSCC.

\section{MATERIALS AND METHODS Clinical trial}

A single institution phase I study was conducted at the Earle A. Chiles Research Institute, Providence Portland Medical Center (PPMC), Providence Cancer Institute. The main eligibility criteria were patients $>18$ years old, Eastern Cooperative Oncology Group (ECOG) performance status 0-2 and advanced MM or HNSCC with measurable or assessable sites of disease. Patients had normal hematologic, hepatic, and renal function before treatment. Exclusion criteria included active infection, need for chronic steroids (above replacement physiological doses) or active autoimmune disease. There were no restrictions for prior systemic therapies or for brain metastases as long as they were stable or improved after local therapy (surgery and/or stereotactic radiation).

After enrollment, patients were assigned consecutively to treatment cohorts. The dose levels of belapectin investigated were 2,4 , and $8 \mathrm{mg} / \mathrm{kg}$ administered IV over $60 \mathrm{~min}$ every 3 weeks $\mathrm{x}$ five doses with infusions completed $60 \mathrm{~min}$ before pembrolizumab infusions. The pembrolizumab dose was fixed at $200 \mathrm{mg}$ IV adtministered over $60 \mathrm{~min}$ every 3 weeks for up to 17 doses. CT or PET CT imaging was obtained at baseline (within 28 days for treatment start), at day 85 and then every 3 months thereafter in patients who continued protocol therapy. Patients with clinical benefit (defined as a CR or PR or SD using RECIST V.1.1 criteria) at day 85 could continue pembrolizumab monotherapy for up to 17 cycles (1 year of therapy) assuming documentation of ongoing response and no DLT. Peripheral blood samples for immune response were obtained on days $1,22,43,64$, and 85 of study participation.

The primary objective of the trial was to determine a safe dose of GR-MD-02 used in combination with pembrolizumab $200 \mathrm{mg}$ IV every 3 weeks. Secondary objectives were exploratory including measuring the response rate to combined therapy with GR-MD-02 and pembrolizumab in patients with MM and HNSCC after disease progression on standard therapy, and to assess the immunological activity of GR-MD-02 in combination with pembrolizumab by measuring CD4+ T cells with a memory phenotype, CD8+ $\mathrm{T}$ cells with effector phenotype, tumor-specific $\mathrm{T}$ cells using autologous and/or HLA-matched tumor when available, examine the composition of the tumor immune infiltrate from tumor biopsies (when feasible).

\section{Flow cytometry}

Whole blood immune profiling assays were conducted as previously described. ${ }^{58}$ Briefly, heparinized whole blood was stained with a cocktail of antibodies to identify T-cell subsets as well as monocytic MDSCs. Stained cells were then incubated with BD FACS lysing solution (BD Biosciences, Franklin Lakes, NJ) followed by washing. For intracellular antigens (eg, Ki-67, granzyme B, and perforin), cells were permeabilized with the Permeabilizing Solution 2 (BD Biosciences) followed by incubation with staining antibodies. Samples were then acquired with a BD LSRFortessa (BD Biosciences). Data were analyzed with FlowJo version 10 (BD Biosciences). The levels of activation (ICOS), proliferation (Ki-67), and functional (granzyme B, perforin) markers were assessed on effector memory (CD45RA ${ }^{-} \mathrm{CCR}^{-}$) CD4+ and CD8+ T-cell subsets (online supplemental figure 4). Monocytic MDSCs were defined as lineage (CD3, CD7, CD19, and CD20) negative, CD15 CD11b+CD33+CD14+HLA-DR ${ }^{\text {low }}$ and PMNMDSCs as lineage (CD3, CD7, CD19, and CD20) negative and $\mathrm{CD} 15+\mathrm{CD} 11 \mathrm{~b}+\mathrm{CD} 33+\mathrm{CD} 14^{-} \mathrm{HLA}^{-D R^{\text {low }}}$ (online supplemental figure 5). The frequency of MDSCs within the lymphocyte plus monocyte gate was used to assess their relationship to treatment effect. Absolute numbers of MDSCs were enumerated using values obtained from complete blood count. 


\section{Multiplex ELISA}

Levels of serum cytokines and chemokines pretreatment and post-treatment were measured in triplicate using a Cytokine/Chemokine/Growth Factor 45-Plex Human ProcartaPlex Panel 1 kit (ThermoFisher, Waltham, MA) and Luminex 200 (Austin, TX).

\section{Multiplex immunohistochemistry (mIHC)}

Five micrometer sections from pretreatment formalinfixed paraffin-embedded tumor biopsies were prepared and stained using the PerkinElmer Opal Kit as previously described.$^{59} \mathrm{mIHC}$ was used to examine the presence and subtypes of leukocytes as well as PD-L1 expression in tumor samples and analyzed by multiplex imaging. Slides were deparaffinized in xylene and alcohol. For fluorescence microscopy, Tris-EDTA pH9.0 was used for retrieving antigens in microwave oven for $15 \mathrm{~min} .3 \% \mathrm{H}_{2} \mathrm{O}_{2}$ was used to block endogenous peroxidase. Slides were blocked with antibody diluent/block (PerkinElmer) before staining with for the following antigens: CD3 (clone SP7; Spring Bioscience), PD-L1 (clone E1L3N; Cell Signaling), CD8 (clone SP16; Spring Bioscience; FoxP3 (clone 236A/E7; Abcam), CD163 (clone MRQ-26; Ventana), galectin-3 (clone 9C4; Cell Marque), SOX10 (BC43; Biocare Medical) for melanoma, and pan-cytokeratin (clone AE1/AE3 clone; DAKO)for HNSCC followed by the antimouse or antirabbit secondary RHRP520G/MHRP520G (Biocare Medical). The signals were amplified using TSA-conjugated OPAL fluorophores (PerkinElmer). Citrate buffer (1X, pH 6.0) was used to strip primary and secondary antibodies and $3 \% \mathrm{H}_{2} \mathrm{O}_{2}$ was used to kill exogenous horseradish peroxide before staining the next marker. Slides were counterstained with DAPI, mounted in Prolong mounting medium (ThermoFisher) and visualized using the Vectra Microscopy imaging system (Akoya Biosciences). Representative regions were captured at 200× magnification using Vectra Software and were analyzed using inForm Image Analysis software (Akoya Biosciences). A total of 3-10 regions of interest per slide were selected for analysis following Pathology review.

\section{Monitoring serum pembrolizumab concentration}

The serum concentration of pembrolizumab was measured by a nano-surface and molecular-orientation limited proteolysis (nSMOL) assay, ${ }^{60} 61$ which uses resin beads containing mAb-capturing Protein A-coated nanopores for the selective cleavage of the Fab region of $\mathrm{mAbs}$ in the presence of trypsin-immobilized nanobeads for detection of signature peptides by a multiple reaction monitoring mass spectrometry (MRM-MS). Briefly, a peptide structure of tryptic pembrolizumab mAb peptides was identified by a high-resolution microflow liquid chromatography-quadrupole time-of-flight MS (Nexera Mikros LC-QTOF, LCMS-9030, Shimadzu, Kyoto, Japan), and fragment ions were assigned using an in-house Mascot server and Distiller with pembrolizumab amino acid sequence information (MatrixScience, London, UK). For nSMOL reactions, pembrolizumab in serum was captured by Protein A resin. Protein A resin was recovered on an Ultrafree filter, washed twice with PBS, and substituted with $25 \mathrm{mM}$ tris- $\mathrm{HCl}(\mathrm{pH} 8.0)$. The nSMOL reaction was carried out by mixing with trypsin-immobilized FG-beads (nSMOL Antibody BA, Shimadzu) with gentle vortexing at $50^{\circ} \mathrm{C}$ for 5 hour in saturated vapor atmosphere. After proteolysis, the reaction was stopped by adding formic acid at a final concentration of $0.5 \%$. The peptide solution was collected and analyzed by MRM-MS using triplequadrupole LCMS-8060 (Shimadzu). The signature peptide LLIYLASYLESGVPAR from pembrolizumab complementarity-determining region (CDR-2, L-chain) and the P14R internal standard were simultaneously quantified with parent $\mathrm{m} / \mathrm{z} 882.6(2+)>$ fragmentm $/ \mathrm{z}$ $343.2(\mathrm{y} 3,1+)$ (retention time $3.4 \mathrm{~min})$.

\section{Reanalysis of bulk RNA-Seq data}

Data set published by Gide $e t a l^{62}$ were downloaded from the SRA (PRJEB23709). We have quantified gene expression (TPM) using salmon-v.0.12.0. ${ }^{63}$ The transcript counts were summarized to gene level using tximport. ${ }^{64}$ Data normalization followed by differential gene expression analysis was carried out using edgeR. ${ }^{65}$

\section{Statistics}

The following statistical analyses were done using Prism V.8 (GraphPad Software; La Jolla, CA) or R V.3.6.3 ( $\mathrm{R}$ Core Team, 2020). The difference between nonresponders and responders for individual markers were evaluated using Wilcoxon rank-sum test or Welch's t-test. To compare the markers between pretreatment and posttreatment, Wilcoxon signed-rank test or paired t-test was used. We further performed Classification and Regression Tree analyses using statistical $\mathrm{R}$ package "rpart", to determine hierarchically how biomarkers fall into homogeneous subgroups, and then evaluated the association of subgroups with response using Fisher exact test. ${ }^{66}$ PFS was calculated from the time of treatment to progression or death. Kaplan-Meier method with log-rank test was used for PFS analyses. A p-value of $<0.05$ was considered significant.

\section{Twitter William L Redmond @WWredmond4}

Acknowledgements We thank Dr Pol F Boudes of Galectin Therapeutics and Dr Walter Urba for critical review of the manuscript.

Contributors $\mathrm{BC}$ made substantial contributions to the conception, design, analysis and interpretation of the data, wrote and conducted the clinical trial, and drafted the manuscript. YK, ASR, NI, TS and ER made substantial contributions to the acquisition, analysis, and interpretation of the data. RSL contributed to the conception, design, and analysis of the study and clinical care of patients. ZS, YW, VR, BB, and IH-M made substantial contributions to the acquisition and analysis of the data. CBF and GM contributed to patient care and clinical data acquisition. SCC provided biostatistical review and support. PGT, EZ, JRH, and HS provided key reagents and contributed to the conception and design of the experiments. WLR made substantial contributions to the conception, design, analysis and interpretation of the data and drafted the manuscript. All authors have reviewed and approved the submitted manuscript.

Funding Funding was provided by the NIH/NCl (R21CA190790), Galectin Therapeutics, and Providence Portland Medical Foundation.

Competing interests WLR: research support from Galectin Therapeutics, Bristol Myers Squibb (BMS), Merck, Tesaro/GlaxoSmithKline, MiNA Therapeutics, 
Inhibrx, Veana Therapeutics, Aeglea Biotherapeutics, Shimadzu, OncoSec, and Calibr. Patents/licensing fees: Galectin Therapeutics. Advisory boards: Nektar Therapeutics, Vesselon. BC: research support from AstraZeneca, Clinigen, and Galectin Therapeutics. Advisory Boards: Replimmune, Clinigen, BMS, and Merck. Leidner: research support from BMS. Advisory boards: Merck, Oncolys, Sanofi, and Regeneron. YW: advisory boards: Roche, Amgen, Alexion, and AstraZeneca. NI and TS: employees of Shimadzu Scientific Instruments. Traber: stockholder of Galectin Therapeutics; employee and stockholder for Selecta Biosciences. EZ, JRH, and HS: employees and stockholders of Galectin Therapeutics. All other authors had no relevant disclosures.

\section{Patient consent for publication Not required.}

Ethics approval Signed informed consent was obtained before enrollment. The study was approved by the Providence Health System Regional Institutional Review Board-Oregon (IRB\#2020000655) and listed on Cancer.gov (NCT02575404).

Provenance and peer review Not commissioned; externally peer reviewed.

Data availability statement All data relevant to the study are included in the article or uploaded as supplementary information.

Supplemental material This content has been supplied by the author(s). It has not been vetted by BMJ Publishing Group Limited (BMJ) and may not have been peer-reviewed. Any opinions or recommendations discussed are solely those of the author(s) and are not endorsed by BMJ. BMJ disclaims all liability and responsibility arising from any reliance placed on the content. Where the content includes any translated material, BMJ does not warrant the accuracy and reliability of the translations (including but not limited to local regulations, clinical guidelines, terminology, drug names and drug dosages), and is not responsible for any error and/or omissions arising from translation and adaptation or otherwise.

Open access This is an open access article distributed in accordance with the Creative Commons Attribution Non Commercial (CC BY-NC 4.0) license, which permits others to distribute, remix, adapt, build upon this work non-commercially, and license their derivative works on different terms, provided the original work is properly cited, appropriate credit is given, any changes made indicated, and the use is non-commercial. See http://creativecommons.org/licenses/by-nc/4.0/.

\section{ORCID iDs}

Brendan D Curti http://orcid.org/0000-0003-3948-2708

Rom S Leidner http://orcid.org/0000-0003-0788-7938

William L Redmond http://orcid.org/0000-0002-2572-1731

\section{REFERENCES}

1 Egen JG, Kuhns MS, Allison JP. Ctla-4: new insights into its biological function and use in tumor immunotherapy. Nat Immunol 2002;3:611-8.

2 Zang X, Allison JP. The B7 family and cancer therapy: costimulation and coinhibition. Clin Cancer Res 2007;13:5271-9.

3 Hodi FS, O'Day SJ, McDermott DF, et al. Improved survival with ipilimumab in patients with metastatic melanoma. $N$ Engl J Med 2010;363:711-23.

4 Wolchok J. How recent advances in immunotherapy are changing the standard of care for patients with metastatic melanoma. Ann Oncol 2012;23 Suppl 8:viii15-21.

5 Robert C, Ribas A, Wolchok JD, et al. Anti-programmed-deathreceptor-1 treatment with pembrolizumab in ipilimumab-refractory advanced melanoma: a randomised dose-comparison cohort of a phase 1 trial. Lancet 2014;384:1109-17.

6 Hamid O, Robert C, Daud A, et al. Safety and tumor responses with lambrolizumab (anti-PD-1) in melanoma. N Engl J Med 2013;369:134-44

7 Duraiswamy J, Kaluza KM, Freeman GJ, et al. Dual blockade of PDand CTLA-4 combined with tumor vaccine effectively restores T-cell rejection function in tumors. Cancer Res 2013;73:3591-603.

8 Yu P, Steel JC, Zhang M, et al. Simultaneous inhibition of two regulatory T-cell subsets enhanced interleukin-15 efficacy in a prostate tumor model. Proc Natl Acad Sci U S A 2012;109:6187-92.

9 Curran MA, Montalvo W, Yagita H, et al. Pd-1 and CTLA-4 combination blockade expands infiltrating $T$ cells and reduces regulatory $\mathrm{T}$ and myeloid cells within $\mathrm{B} 16$ melanoma tumors. Proc Natl Acad Sci U S A 2010;107:4275-80.

10 McNamara MJ, Hilgart-Martiszus I, Barragan Echenique DM, et al. Interferon- $\gamma$ Production by Peripheral Lymphocytes Predicts Survival of Tumor-Bearing Mice Receiving Dual PD-1/CTLA-4 Blockade. Cancer Immunol Res 2016;4:650-7.
11 Larkin J, Chiarion-Sileni V, Gonzalez R, et al. Combined nivolumab and ipilimumab or monotherapy in untreated melanoma. $N$ Engl J Med 2015;373:23-34.

12 Tang J, Pearce L, O'Donnell-Tormey J, et al. Trends in the global immuno-oncology landscape. Nat Rev Drug Discov 2018;17:783-4.

13 Yang R-Y, Rabinovich GA, Liu F-T. Galectins: structure, function and therapeutic potential. Expert Rev Mol Med 2008;10:e17.

14 Wang Y-G, Kim S-J, Baek J-H, et al. Galectin-3 increases the motility of mouse melanoma cells by regulating matrix metalloproteinase-1 expression. Exp Mol Med 2012;44:387-93.

15 Braeuer RR, Zigler M, Kamiya T, et al. Galectin-3 contributes to melanoma growth and metastasis via regulation of NFAT1 and autotaxin. Cancer Res 2012;72:5757-66.

16 Mourad-Zeidan AA, Melnikova VO, Wang H, et al. Expression profiling of Galectin-3-depleted melanoma cells reveals its major role in melanoma cell plasticity and vasculogenic mimicry. Am J Pathol 2008;173:1839-52.

17 MacKinnon AC, Farnworth SL, Hodkinson PS, et al. Regulation of alternative macrophage activation by galectin-3. J Immunol 2008;180:2650-8.

18 Hsu DK, Chen H-Y, Liu F-T. Galectin-3 regulates T-cell functions. Immunol Rev 2009;230:114-27.

19 Farhad M, Rolig AS, Redmond WL. The role of galectin-3 in modulating tumor growth and immunosuppression within the tumor microenvironment. Oncoimmunology 2018;7:e1434467.

20 Capalbo C, Scafetta G, Filetti M, et al. Predictive biomarkers for checkpoint inhibitor-based immunotherapy: the galectin-3 signature in NSCLCs. Int J Mol Sci 2019;20:1607. doi:10.3390/ ijms20071607

21 Demotte N, Wieërs G, Van Der Smissen P, et al. A galectin-3 ligand corrects the impaired function of human CD4 and CD8 tumorinfiltrating lymphocytes and favors tumor rejection in mice. Cancer Res 2010;70:7476-88.

22 Vuong L, Kouverianou E, Rooney CM, et al. An orally active galectin-3 antagonist inhibits lung adenocarcinoma growth and augments response to PD-L1 blockade. Cancer Res 2019;79:1480-92.

23 Chalasani N, Abdelmalek MF, Garcia-Tsao G, et al. Effects of Belapectin, an inhibitor of galectin-3, in patients with nonalcoholic steatohepatitis with cirrhosis and portal hypertension. Gastroenterology 2020;158:1334-45.

24 Ritchie S, Neal D, Shlevin H, et al. A phase 2A, open-label pilot study of the galectin-3 inhibitor GR-MD-02 for the treatment of moderate-to-severe plaque psoriasis. J Am Acad Dermatol 2017;77:753-5.

25 Harrison SA, Marri SR, Chalasani N, et al. Randomised clinical study: GR-MD-02, a galectin-3 inhibitor, vs. placebo in patients having nonalcoholic steatohepatitis with advanced fibrosis. Aliment Pharmacol Ther 2016;44:1183-98.

26 Baecher-Allan C, Wolf E, Hafler DA. Mhc class II expression identifies functionally distinct human regulatory T cells. J Immunol 2006;176:4622-31.

27 Traber PG, Zomer E. Therapy of experimental NASH and fibrosis with galectin inhibitors. PLoS One 2013;8:e83481.

28 Novak R, Dabelic S, Dumic J. Galectin-1 and galectin-3 expression profiles in classically and alternatively activated human macrophages. Biochim Biophys Acta 2012;1820:1383-90.

29 Bronte V, Brandau S, Chen S-H, et al. Recommendations for myeloid-derived suppressor cell Nomenclature and characterization standards. Nat Commun 2016;7:12150.

30 Kumar V, Patel S, Tcyganov E, et al. The nature of myeloid-derived suppressor cells in the tumor microenvironment. Trends Immunol 2016;37:208-20.

31 Marvel D, Gabrilovich DI. Myeloid-derived suppressor cells in the tumor microenvironment: expect the unexpected. J Clin Invest 2015;125:3356-64.

32 Ostrand-Rosenberg S, Fenselau C. Myeloid-Derived suppressor cells: immune-suppressive cells that impair antitumor immunity and are sculpted by their environment. J Immunol 2018;200:422-31.

33 Franciszkiewicz K, Boissonnas A, Boutet M, et al. Role of chemokines and chemokine receptors in shaping the effector phase of the antitumor immune response. Cancer Res 2012;72:6325-32.

34 Lu S, Stein JE, Rimm DL, et al. Comparison of biomarker modalities for predicting response to PD-1/PD-L1 checkpoint blockade: a systematic review and meta-analysis. JAMA Oncol 2019. doi:10.1001/jamaoncol.2019.1549. [Epub ahead of print: $18 \mathrm{Jul}$ 2019].

35 Yeong J, Tan T, Chow ZL, et al. Multiplex immunohistochemistry/ immunofluorescence (mlHC/IF) for PD-L1 testing in triple-negative breast cancer: a translational assay compared with conventional IHC. J Clin Pathol 2020;73:557-62. 
36 Kansy BA, Concha-Benavente F, Srivastava RM, et al. PD-1 Status in $\mathrm{CD}^{+}{ }^{+}$Cells Associates with Survival and Anti-PD-1 Therapeutic Outcomes in Head and Neck Cancer. Cancer Res 2017;77:6353-64.

37 Huang AC, Orlowski RJ, Xu X, et al. A single dose of neoadjuvant PD-1 blockade predicts clinical outcomes in resectable melanoma. Nat Med 2019;25:454-61.

38 Fairfax BP, Taylor CA, Watson RA, et al. Peripheral CD8 ${ }^{+} \mathrm{T}$ cell characteristics associated with durable responses to immune checkpoint blockade in patients with metastatic melanoma. Nat Med 2020;26:193-9.

39 Wu TD, Madireddi S, de Almeida PE. Peripheral T cell expansion predicts tumour infiltration and clinical response. Nature 2020;579:274-8.

40 Turner DC, Kondic AG, Anderson KM, et al. Pembrolizumab exposure-response assessments challenged by association of cancer cachexia and catabolic clearance. Clin Cancer Res 2018;24:5841-9.

41 Basak EA, Koolen SLW, Hurkmans DP, et al. Correlation between nivolumab exposure and treatment outcomes in non-small-cell lung cancer. Eur J Cancer 2019;109:12-20.

42 Ribas A, Puzanov I, Dummer R, et al. Pembrolizumab versus investigator-choice chemotherapy for ipilimumab-refractory melanoma (KEYNOTE-002): a randomised, controlled, phase 2 trial. Lancet Oncol 2015;16:908-18.

43 Schachter J, Ribas A, Long GV, et al. Pembrolizumab versus ipilimumab for advanced melanoma: final overall survival results of a multicentre, randomised, open-label phase 3 study (KEYNOTE-006). Lancet 2017;390:1853-62.

44 Seiwert TY, Burtness B, Mehra R, et al. Safety and clinical activity of pembrolizumab for treatment of recurrent or metastatic squamous cell carcinoma of the head and neck (KEYNOTE-012): an open-label, multicentre, phase 1B trial. Lancet Oncol 2016;17:956-65.

45 Ferris RL, Blumenschein G, Fayette J, et al. Nivolumab for recurrent squamous-cell carcinoma of the head and neck. N Engl J Med 2016;375:1856-67.

46 Burtness B, Harrington KJ, Greil R, et al. Pembrolizumab alone or with chemotherapy versus cetuximab with chemotherapy for recurrent or metastatic squamous cell carcinoma of the head and neck (KEYNOTE-048): a randomised, open-label, phase 3 study. Lancet 2019;394:1915-28.

47 Cohen EEW, Soulières D, Le Tourneau C, et al. Pembrolizumab versus methotrexate, docetaxel, or cetuximab for recurrent or metastatic head-and-neck squamous cell carcinoma (KEYNOTE-040): a randomised, open-label, phase 3 study. Lancet 2019;393:156-67.

48 Robert C, Schachter J, Long GV, et al. Pembrolizumab versus ipilimumab in advanced melanoma. N Engl J Med 2015;372:2521-32.

49 Kamphorst AO, Pillai RN, Yang S, et al. Proliferation of PD-1+ CD8 T cells in peripheral blood after PD-1-targeted therapy in lung cancer patients. Proc Natl Acad Sci U S A 2017;114:4993-8.

50 Mazzaschi G, Minari R, Zecca A, et al. Soluble PD-L1 and circulating CD8+PD-1+ and NK cells enclose a prognostic and predictive immune effector score in immunotherapy treated NSCLC patients. Lung Cancer 2020;148:1-11.
51 Hernandez C, Arasanz H, Chocarro L, et al. Systemic blood immune cell populations as biomarkers for the outcome of immune checkpoint inhibitor therapies. Int J Mol Sci 2020;21:2411.

52 Meyer C, Cagnon L, Costa-Nunes CM, et al. Frequencies of circulating MDSC correlate with clinical outcome of melanoma patients treated with ipilimumab. Cancer Immunol Immunother 2014:63:247-57.

53 Retseck J, Nasr A, Lin Y, et al. Long term impact of CTLA4 blockade immunotherapy on regulatory and effector immune responses in patients with melanoma. J Transl Med 2018;16:184.

54 Weber J, Gibney G, Kudchadkar R, et al. Phase I/II study of metastatic melanoma patients treated with nivolumab who had progressed after ipilimumab. Cancer Immunol Res 2016;4:345-53.

55 Hurkmans DP, Basak EA, van Dijk T, et al. A prospective cohort study on the pharmacokinetics of nivolumab in metastatic non-small cell lung cancer, melanoma, and renal cell cancer patients. J Immunother Cancer 2019;7:192.

56 Coss CC, Clinton SK, Phelps MA. Cachectic cancer patients: immune to checkpoint inhibitor therapy? Clin Cancer Res 2018;24:5787-9.

57 Bins S, Koolen SLW, Mathijssen RHJ. Pembrolizumab exposureresponse assessments challenged by association of cancer cachexia and catabolic Clearance-Letter. Clin Cancer Res 2019;25:3192.

58 Koguchi Y, Gonzalez IL, Meeuwsen TL, et al. A semi-automated approach to preparing antibody cocktails for immunophenotypic analysis of human peripheral blood. J Vis Exp 2016;108:e53485.

59 Baird JR, Bell RB, Troesch V, et al. Evaluation of explant responses to sting ligands: personalized Immunosurgical therapy for head and neck squamous cell carcinoma. Cancer Res 2018;78:6308-19.

60 Iwamoto N, Shimada T. Recent advances in mass spectrometrybased approaches for proteomics and biologics: great contribution for developing therapeutic antibodies. Pharmacol Ther 2018;185:147-54.

61 Iwamoto N, Shimada T, Umino Y, et al. Selective detection of complementarity-determining regions of monoclonal antibody by limiting protease access to the substrate: nano-surface and molecular-orientation limited proteolysis. Analyst 2014;139:576-80.

62 Gide TN, Quek C, Menzies AM, et al. Distinct immune cell populations define response to anti-PD-1 monotherapy and AntiPD-1/Anti-CTLA-4 combined therapy. Cancer Cell 2019;35:238-55.

63 Patro R, Duggal G, Love Ml, et al. Salmon provides fast and bias-aware quantification of transcript expression. Nat Methods 2017;14:417-9.

64 Soneson C, Love MI, Robinson MD. Differential analyses for RNASeq: transcript-level estimates improve gene-level inferences. F1000Res 2015;4:1521.

65 Robinson MD, McCarthy DJ, Smyth GK. edgeR: a Bioconductor package for differential expression analysis of digital gene expression data. Bioinformatics 2010;26:139-40.

66 Barlin JN, Zhou Q, St Clair CM, et al. Classification and regression tree (CART) analysis of endometrial carcinoma: seeing the forest for the trees. Gynecol Oncol 2013;130:452-6. 